\title{
Correlation between Sagittal Morphology of Lower Lumbar Endplate and Degenerative Changes in Patients with Lumbar Disc Herniation
}

\section{Chongqing Xu ( $\nabla 09663990 @ q q . c o m)$}

Long Hua Hospital https://orcid.org/0000-0001-9654-793X

Mengchen Yin

Long Hua Hospital

\section{Wen Mo}

Long Hua Hospital

\section{Research article}

Keywords: sagittal morphology, lumbar endplate, lumbar disc herniation, magnetic resonance imaging

Posted Date: August 13th, 2020

DOI: https://doi.org/10.21203/rs.3.rs-56740/v1

License: (c) (i) This work is licensed under a Creative Commons Attribution 4.0 International License.

Read Full License 


\section{Abstract}

Background: As an important anatomic factor in the process of Lumbar disc herniation (LDH), the correlation between endplate sagittal morphology and intervertebral disc degeneration (IDD) is unclear. and research on imaging data of lumbar endplate in patients with LDH is still insufficient. Our study aimed to observe the morphological change of the lower lumbar endplate (L3-S1) in patients with LDH on magnetic resonance imaging (MRI), and analyze its correlation with the degree of IDD.

Methods/Design: All 116 patients were included. Based on their MRI, we divided endplates into three types (concave, flat and irregular), assigned intervertebral discs with Grade I-V given 1-5 points successively according to Pfirrmann system, and determined whether there was Modic change of each endplate. The correlation between the morphology of endplate and the degree of IDD was analyzed.

Results: There were excellent inter-observer agreement for each item we analyzed (ICC > 0.75). Concave endplate appeared most frequently $(187,53.7 \%)$ and mainly distributed in $L 3 / 4$ and $L 4 / 5$, while irregular endplate was the least common type $(54,15.5 \%)$ and mainly concentrated in L5/S1. The IDD degree of corresponding disc increased gradually from concave $(3.27 \pm 0.81)$ to irregular endplates $(4.25 \pm 0.79)(P$ $<0.05)$. Irregular endplates were more likely to have Modic changes than concave and flat endplates $(P<$ $0.05)$.

Conclusion: The sagittal morphology of lower lumbar endplate is related to Modic changes and degree of IDD (based on Pfirrmann grading system) in patients with LDH, and the concave endplate mostly reflects a lower degree of lumbar disc degeneration, which has substantial clinical significance.

\section{Background}

Lumbar disc herniation (LDH) is an usual type of lumbar degeneration disease, which is a common cause of low back pain and lower extremity dysfunction. Studies indicate that aging, obesity, smoking, endplate injury, lumbar rotation load and bone mineral density are risk factors for LDH.[1,2] The endplate is closely adjacent to the intervertebral disc, which is the direct conduction site of disc biomechanics. The mechanism of spinal compensation is complex, and the current cognition is still limited. Clinical studies have shown that the endplate will remodel to adapt to intervertebral disc degeneration (IDD),[3] and imaging changes of endplate are often observed in IDD.[4] With the development of surgical procedures, devices in some technologies such as disc replacement and interbody fusion require concave endplate designs to make the implant firmer, and better accord with the morphological variability and biomechanical demand in vivo.[5,6] However, as an important anatomic factor in the process of LDH, the correlation between endplate sagittal morphology and IDD is unclear, and research on imaging data of lumbar endplate in patients with LDH is still insufficient. Because of the special biomechanical environment of the lower lumbar disc, it is more prone to degeneration than the upper lumbar spine.[7] Therefore, our study aims to observe the morphological and signal changes of the lower lumbar endplate 
(L3-S1) in patients with LDH on magnetic resonance imaging (MRI), analyze the relationship between the morphology of the endplate and the degree of IDD, and explore its clinical significance.

\section{Materials And Methods}

\subsection{Patient Case Selection and Evaluation}

We obtained institutional review board approval from our ethics committee to perform this study, and informed signed consents were provided by all participating subjects. Database records of patients with lower LDH (L3-S1) treated in our hospital were retrospectively collected and analyzed between January 1 , 2019 , to December 31, 2019. Patients included in the study were required to have available clinical data and should have performed lumbar MRI. A complete MRI examination must include T1- and T2-weighted turbo spin sagittal images without fat suppression to cover all types of Pfirrmann grading system.[8] MRI data were gained through 1.5-T whole-body imaging system. Exclusion criteria were as follows: 1) Patients with a history of tumor, infection, fracture, deformity, spondylolisthesis, ankylosing spondylitis, rheumatism, osteoporosis or other metabolic conditions that may affect the morphology of lumbar endplate; 2) Patients with previous spinal surgery history. According to the criteria, a total of 116 consecutive patients were involved.

The degree of IDD was judged and scored according to the Pfirrmann grading system[8] which was divided into five grades, with Grade I to $V$ given 1-5 points successively. Based on the classification of Pappou et al.,[7] the shape of endplate was divided into concave, flat and irregular levels (Figure 1). When the upper and lower endplates of the same segment had two types of shape, the endplates of the same segment were classified as the severer type. In addition, we determined whether there was Modic change of each endplate according to the Modic classification (Figure 2).[9]

\subsection{Statistical analysis}

1 spine surgeon and 1 radiologist were selected to be evaluators while they did not know the identity of the patient, the medical history of them, and the original classification used in clinical care. Each evaluator was provided necessary original literature and relevant information to assess cases. In case of controversy, they would discuss until they come to a consensus. If not, another spine surgeon would be asked to assess, and the result of majority approval would be taken.

All data analyses were performed using Statistical Packages of Social Sciences (SPSS) software (version 22.0). Measurement data are expressed as mean \pm standard deviation, and ordinal data are expressed as percentages. The inter-observer reliability of the evaluations were tested through interclass correlation coefficient (ICC),[10] and the values were expressed with a 95\% confidence interval (CI). The larger value represented the better agreement. There were three levels of agreement for ICC, with 0.00 to 0.40 considered poor agreement, 0.40 to 0.74 fair to good agreement, and 0.75 to 1.00 excellent agreement. $[11,12]$ The correlation between endplate shape and degree of IDD was analyzed by rank sum test, while the correlation between endplate shape and Modic change, endplate shape and disc herniation was 
analyzed by chi-square test. Meanwhile, $p$ values of $<0.05$ were considered statistically significant for all the above.

\section{Result}

A total of 116 consecutive cases were involved in our study, including 47 males and 69 females with a mean age of $52.6 \pm 8.4$ years (range from 25 to 67 years). There were totally 348 discs and 348 pairs of endplates (L3/4, L4/5, L5/S1) taken into consideration by each evaluator (Table 1).

Table 1. General Information of the Patients

\begin{tabular}{clc}
\hline No. & Clinical Information & Number \\
\hline & & \\
$\mathbf{1}$ & Sex (Male/Female) & $47 / 69$ \\
$\mathbf{2}$ & Mean age (years) & $52.6 \pm 8.4$ \\
$\mathbf{3}$ & Herniated/Non-herniated discs & $158 / 190$ \\
$\mathbf{4}$ & Segment of herniation & \\
& L3/4 & 39 \\
& L4/5 & 65 \\
& L5/S1 & 54 \\
\hline
\end{tabular}

\subsection{Inter-observer Reliability}

Based on reliability analysis of the results, the inter-observer agreement of endplate shape, Pfirrmann classification, Modic change and disc herniation on MRI images were excellent, ICC values of each item were calculated as follows: $0.895,0.766,0.854$, and 0.923 . The results were considered without significant difference $(p>0.05)$ (Table 2).

Table 2. Inter-observer Reliability of Each Item between Evaluators

\begin{tabular}{lcc}
\hline Items & \multicolumn{2}{c}{ Inter-observer Reliability } \\
\cline { 2 - 3 } & ICC & $p$ \\
\hline Endplate morphology & 0.895 & $0.507^{*}$ \\
Pfirrmann classification & 0.766 & $0.251^{*}$ \\
Modic change & 0.854 & $0.489^{*}$ \\
Disc herniation & 0.923 & $0.762^{*}$ \\
\hline
\end{tabular}

*There was no significant difference between the 2 evaluators for the item.

\subsection{Measurements and Correlations}


Among the three types of morphology, concave endplate appeared most frequently $(187,53.7 \%)$ and mainly distributed in L3/4 and L4/5 segments; There were 107 (30.8\%) pairs of flat endplates, which increased gradually from L3/4 to L5/S1; Irregular endplate was the least common type $(54,15.5 \%)$ and mainly concentrated in L5/S1 (Table 3).

Table 3. The Morphology of Endplates in Different Segments of All Patients

\begin{tabular}{lcccc}
\hline Morphology & Number (\%) & \multicolumn{3}{c}{ Endplate Segment } \\
\cline { 3 - 5 } & & L3/4 & L4/5 & L5/S1 \\
\hline Concave & $187(53.7 \%)$ & 97 & 69 & 21 \\
Flat & $107(30.8 \%)$ & 13 & 24 & 70 \\
Irregular & $54(15.5 \%)$ & 10 & 12 & 32 \\
\hline
\end{tabular}

According to the Pfirrmann grading system, the degeneration degree of corresponding intervertebral disc increased gradually from concave $(3.27 \pm 0.81)$ to irregular endplates $(4.25 \pm 0.79)(p<0.05)$, and IDD of Grade IV accounted for the most. In addition, the proportion of Grade V in irregular endplate is far greater than that of the other two types, as shown in Table 4.

Table 4. The Disc Degeneration Grade and Score of Patients Based on Pfirrmann Classification

\begin{tabular}{|c|c|c|c|c|c|c|}
\hline \multirow[t]{2}{*}{ Morphology } & \multicolumn{5}{|c|}{ Grade of Pfirrmann Classification } & \multirow{2}{*}{ Scores of the Grade (Mean \pm SD) } \\
\hline & $\mathrm{I}$ & II & III & IV & $\mathrm{V}$ & \\
\hline Concave & 4 & 37 & 51 & 94 & 1 & $3.27 \pm 0.81$ \\
\hline Flat & 0 & 8 & 25 & 70 & 4 & $3.84 \pm 0.67 *$ \\
\hline Irregular & 0 & 0 & 2 & 33 & 19 & $4.25 \pm 0.79 * \dagger$ \\
\hline
\end{tabular}

*Compared with concave, $p \llbracket 0.05 .{ }^{\dagger}$ Compared with flat, $p \llbracket 0.05$.

The flat and irregular endplates were more common than the concave ones in the herniated segments ( $p$ $<0.05)$, while there was no significant difference between the flat and irregular types $(p>0.05)$. On the contrary, the concave endplates appeared most frequently in the non-herniated segments, following by the flat ones, with significant difference $(p<0.05)$. There were 49 segments of endplates ( 43 in herniated segment) in 37 patients accompanied by Modic changes. Those endplates in herniated segments accompanied by Modic changes mainly belonged to irregular type, with statistical difference $(p<0.05)$, while there was no significant difference between the concave and flat type $(p>0.05)$ (Table 5).

Table 5. The Relationship among Endplate Morphology, Disc Herniation and Modic Changes of Patients

\begin{tabular}{lccc|ccc}
\hline Morphology & \multicolumn{3}{c|}{ Disc Herniation } & \multicolumn{3}{c}{ No Disc Herniation } \\
\cline { 2 - 7 } & Number & Modic Change & No Modic Change & Number & Modic Change & No Modic Change \\
\hline \multirow{2}{*}{ Concave } & 24 & 5 & 19 & 163 & 2 & 161 \\
Flat & $41^{*}$ & 9 & $32^{*}$ & $66^{*}$ & 1 & $65^{*}$ \\
Irregular & $44^{*}$ & $29^{* \dagger}$ & $15^{\dagger}$ & $10^{* \dagger}$ & 3 & $7^{* \dagger}$ \\
\hline
\end{tabular}


${ }^{*}$ Compared with concave, $\mathrm{p} \square 0.05 .{ }^{\dagger}$ Compared with flat, $\mathrm{p} \square 0.05$.

\section{Discussion}

As a key component of spine structure, endplate plays an important role in nourishing intervertebral disc and conducting stress, and has a significant impact on physiological state and pathological changes of intervertebral disc. In the treatment of IDD, motion preservation techniques are increasingly used. However, these techniques have certain requirements for endplate shape and height of intervertebral space. Therefore, it is helpful to study the correlation between morphology of endplate and IDD in clinical work. Previous studies on the morphology of the endplate mostly focused on the anatomical structure or the use of imaging to observe the transverse and sagittal diameter, circumference and area of endplates, as well as the measurement of their concave angle and relative curvature.[13] Although measuring the concave angle can objectively and quantitatively reflect the depression degree of endplate, it is so complex, and the uncertainty of the arc vertex positioning makes larger deviations. By contrast, the method used in this study can intuitively define the shape of endplate, which is simple to operate.

Harrington et al.[14] observed the influence of axial shape of the endplates on herniated discs, and pointed out that the endplate morphology with high curvature is an independent risk factor for LDH. Pappou et al.[7] analyzed the correlation between endplate morphology and IDD in patients with low back pain. Though their results were similar to ours, the study subjects were different, and they didn't take Modic changes and disc herniation into consideration.

According to our results, we found that concave endplate is the main type in intervertebral discs with lower grade of degeneration on MRI. The biomechanical studies $[15,16]$ show that the stress conducted by normal disc is mainly concentrated in the center of the vertebral endplate. The stress not only affects the volume and shape of the disc, but also has an effect on the endplate. The load of axial stress, especially, can lead to the deformation of endplate and trabecula under it. That may explain why concave endplate is commonly seen in discs with milder IDD. In addition, when the intervertebral disc degenerates, the hydrodynamic characteristics of the nucleus pulposus gradually disappear, and the stress shifts from the center of the endplate to the periphery, resulting in relatively concentrated stress on the peripheral endplate, which not only increases the shear force and makes it prone to microfracture,[15] but also activate the bone reconstruction process of the endplate and vertebral body,[3] which will eventually lead to the gradual loss of the peripheral height of vertebral body and the flattening of endplate.[17] Meanwhile, the stress load of the peripheral part of lumbar intervertebral disc exceeds the normal range, which will lead to the injury of annulus fibrosus and accelerate the occurrence of LDH. Moreover, studies have shown that the pressure of lumbar endplate increases gradually from top to bottom.[18] In our study, with the descent of lumbar segments, the concave endplates gradually decreased, and the flat endplates were increasingly common. The endplates of L5/S1 segment were mainly flat type, which may be related to the higher stress load of L4/5 and L5/S1 segments. The proportion of irregular type was the least and mainly concentrated in L5/S1 segment. We believed that the long-term effect of large stress load may easily lead to the irregular shape of endplate. Since L5/S1 segment is located at the 
lumbosacral junction and has high shear force, irregular endplates are more likely to appear in this segment than in others. In the process of disc herniation, the endplate morphology changes from concave to flat and then irregular, and the degeneration degree of corresponding disc gets increasingly severe. Meanwhile the herniated discs are mainly accompanied with flat and irregular endplates. The result that the proportion of Grade V IDD in irregular endplate is far greater than that of the other two types can also explain this.

Modic changes in MRI signal of endplate are highly correlated with IDD[19] account for approximately $19 \%-59 \%$ of disc degenerative diseases.[20,21] At present, it is generally believed that abnormal stress load after IDD,[4] lumbar instability[22] and release of inflammatory factors[23] will cause vertebral microfracture and affect local microenvironment of marrow, leading to histological changes which ultimately manifest as changes in MRI signal of endplate. Based on our results, Modic changes accounted for $30 \%$ in patients with $\mathrm{LDH}$, and irregular endplates had more Modic changes than the ones of other two types. It is reasonable for us to speculate that the Modic change may be the result of the endplate subjected to long-term abnormal stress and degeneration to a certain extent.

The current study has several limitations. Firstly, limited by the hardware conditions of our radiology department, we didn't use computed tomography (CT) reconstruction which was better for evaluating the sagittal morphology of endplate than MRI. Studies have shown that the bony edge of endplate is more easily identified on CT images.[24] Secondly, the relatively few evaluators. Considering the cognitive differences among specialties, we selected physicians from orthopedics and radiology department to assess the data. Nevertheless, increasing the number of evaluators will eliminate coincidence as much as possible, and improve the reliability of the results. Finally, we didn't analyze the coronal morphology of endplate, and its effect on LDH or other lumbar degeneration disease needs further research. Therefore, high-quality, large sample, and multicenter studies should be performed in our future clinical work to provide spine surgeons with the best evidence-based information.

\section{Conclusion}

The sagittal morphology of lower lumbar endplate is related to Modic changes and degree of IDD (based on Pfirrmann grading system) in patients with LDH, and the concave endplate mostly reflects a lower degree of lumbar disc degeneration, which has substantial clinical significance. However, larger sample and multicenter studies should be performed to improve the accuracy and reliability of the finding, and further clinical correlation should be evaluated in future works.

\section{Abbreviations}

LDH (lumbar disc herniation)

IDD (intervertebral disc degeneration)

MRI (magnetic resonance imaging) 
SPSS (Statistical Packages of Social Sciences)

ICC (interclass correlation coefficient)

$\mathrm{Cl}$ (confidence interval)

CT (computed tomography)

\section{Declarations}

\section{Ethics approval and consent to participate}

The case was reviewed by the Longhua Hospital Ethics Committee and ethical approval was waived as written consent was obtained from the patient.

\section{Consent for publication}

Written patient consent was obtained for publication of all aspects of the case including personal and clinical details and images, which may compromise anonymity.

\section{Availability of data and material}

All supporting data can be provided upon request to the authors.

\section{Competing interests}

All authors read and approved the final manuscript and declare that they have no competing interests.

\section{Funding}

No funding was obtained for this study.

\section{Authors' contributions}

XCQ and YMC are co-first authors of this manuscript. XCQ designed the study and collected the data. YMC did the data analysis. XCQ wrote the manuscript. MW revised the manuscript and decided to submit the manuscript for publication. All authors read and approved the final manuscript.

\section{Acknowledgements}

I want to thank my love, Du Ying, no matter how difficult it is, she never gives up, always cares for me, silently supports me, and gives me courage when I lose confidence. Without her help, understanding, tolerance and support, I believe that the life of a $\mathrm{PhD}$ in these three years will be very different.

\section{References}


1. Li Y, Lord E, Cohen Y, et al. Effects of sagittal endplate shape on lumbar segmental mobility as evaluated by kinetic magnetic resonance imaging. Spine(Phila Pa 1976). 2014;39:E1035-1041.

2. Samartzis D, Karppinen J, Mok F, et al. A population-based study of juvenile disc degeneration and its association with overweight and obesity, low back pain, and diminished functional status. J Bone Joint Surg Am. 2011;93:662-670.

3. Grosland NM, Goel VK. Vertebral endplate morphology followsbone remodeling principles. Spine. 2007;32:667-673.

4. Modic MT. Modic type 1 and type 2 changes. J Neurosurg Spine. 2007;6:150-151.

5. Lakshmanan P, Purushothaman B, Dvorak V, et al. Sagittal endplate morphology of the lower lumbar spine. Eur Spine J. 2012;21:S160-164.

6. Gamradt SC, Wang JC. Lumbar disc arthroplasty. Spine J. 2005;5:95-103.

7. Pappou IP, Cammisa FP Jr, Girardi FP. Correlation of endplate shape on MRI and disc degeneration in surgically treated patients with degenerative disc disease and herniated nucleus pulposus. Spine J. 2007;7:32-38.

8. Pfirrmann CW, Metzdorf A, Zanetti M, et al. Magnetic resonance classification of lumbar intervertebral disc degeneration. Spine. 2001; 26:1873-1878.

9. Modic MT, Masaryk TJ, Ross JS, et al. Imaging of degenerative disk disease. Radiology. 1988;168:177-186.

10. Shrout PE, Fleiss JL. Intraclass correlations: uses in assessing rater reliability. Psychol Bull. 1979;86:420-428.

11. Fleiss J. The design and analysis of clinical experiments. Wiley, New York. 1986;pp 1-31.

12. Landis JR, Koch GG. The measurement of observer agreement for categorical data. Biometrics. 1977;33:159-174.

13. Gu HS, Zhou WY, Li ZY. Clinic research between vertebral endplate morphology and lumbar disc herniation. J Clin Reh Tis Eng Res. 2010;14:9497-9500.

14. Harrington JF, Sungarian A, Rogg J, et al. The relation between vertebral endplate shape and lumbar disc herniations. Spine(Phila Pa 1976). 2001;26:2133-2138.

15. Li FC, Zhang N, Chen WS, et al. Endplate degeneration may be the origination of the vacuum phenomenon in intervertebral discs. Med Hypotheses. 2010;75:169-171.

16. Zhao F, Pollintine P, Hole BD, et al. Discogenic origins of spinal instability. Spine. 2005;30:2621-2630.

17. He X, Liang A, Gao W, et al. The relationship between concave angle of vertebral endplate and lumbar intervertebral disc degeneration. Spine (Phila Pa 1976). 2012. 37:E1068-1073.

18. Li ZG, Zheng LJ, Li GC, et al. Biomechanical research on the properties of the lumbosacral endplates. Chinese Journal of Spine and Spinal Cord. 2007. 17:210-213.

19. Albert HB, Briggs AM, Kent P, et al. The prevalence of MRI-defined spinal pathoanatomies and their association with Modic changes in individuals seeking care for low back pain. Eur Spine J. 2011;20:1355-1362. 
20. Kjaer P, Leboeuf-Yde C, Korsholm L, et al. Magnetic resonance imaging and low back pain in adults: a diagnostic imaging study of 40-year-old men and women. Spine. 2005;30:1173-1180.

21. Kuisma M, Karppinen J, Niinimäki J, et al. Modic changes in endplates of lumbar vertebral bodies: prevalence and association with low back and sciatic pain among middle-aged male workers. Spine. 2007;32:1116-1122.

22. Zhang YH, Zhao CQ, Jiang LS, et al. Modic changes: a systematic review of the literature[J]. Eur Spine J. 2008;17:1289-1299.

23. Peng $B$, Zhang $Y$, Hou $S$, et al. Intradiscal methylene blue injection for the treatment of chronic discogenic low back pain. Eur Spine J. 2007;16:33-38.

24. Rajasekaran S, Bajaj N, Tubaki V, et al. ISSLS Prize winner: The anatomy of failure in lumbar disc herniation: an in vivo, multimodal, prospective study of 181 subjects. Spine (Phila Pa 1976). 2013;38:1491-1500.

\section{Figures}

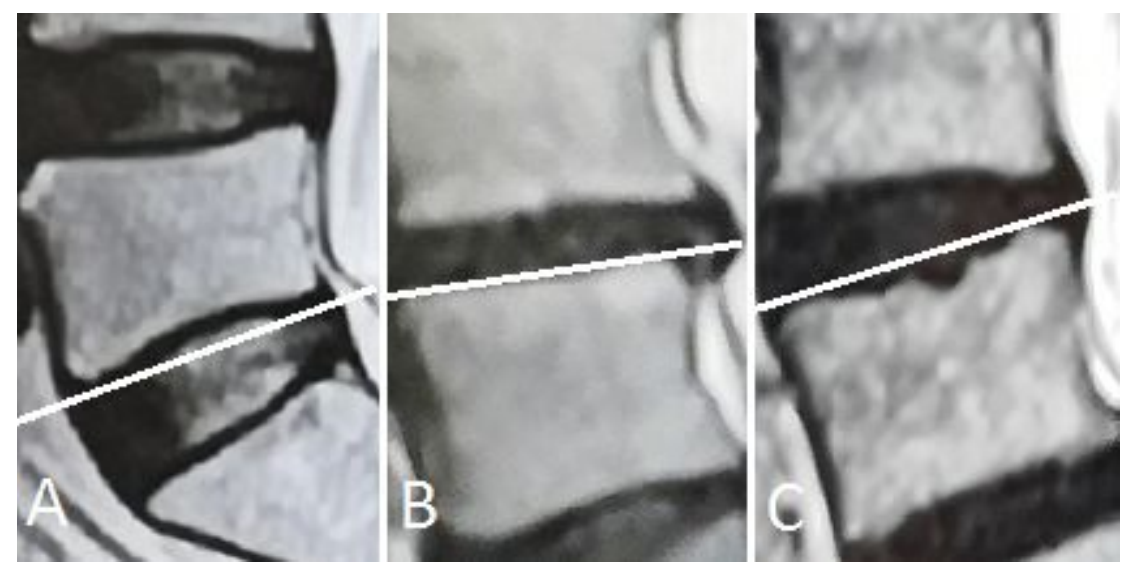

Figure 1

Endplate shapes on sagittal T2-weighed MRI. Concave(A), flat(B), and irregular(C) 

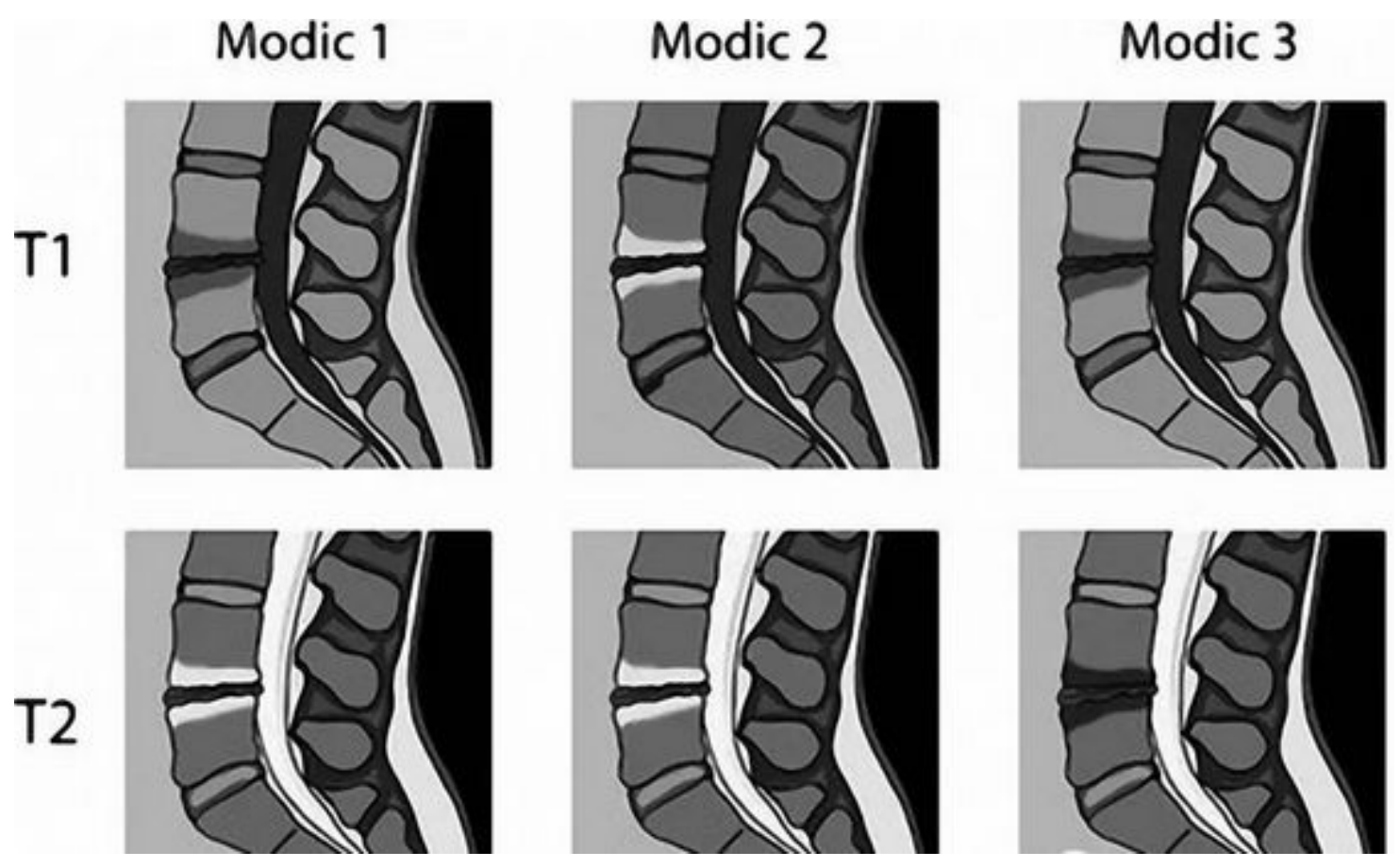

Figure 2

Modic changes on T1- and T2-weighed MRI 